\title{
SOME CHARACTERIZATIONS OF DISCRETE UNIMODALITY
}

\author{
Emile M.J. BERTIN \\ Department of Mathematics. University of Utrecht. Budapestlaan 6. 3508 TA Uirecht. The Nerherlands
}

\section{Radu THEODORESCU}

Department of Mathematics, Laval University, Quebec, Que., Canada GIK 7P4

Received January 1983

Revised May 1983

\begin{abstract}
Let $F$ be a discrete distribution function on $\mathbb{Z}$. This paper gives a characterization of discrete unimodal distribution functions (Theorem 5.1) and a representation theorem for those distribution functions (Theorem 6.3), both in terms of their Lévy concentration functions.
\end{abstract}

AMS 1979 Subject Classification: Primary 60E05, 62E10; Secondary 26A51.

Keywords and phrases: discrete unimodality, discrete distribution function, unimodal distribution function, mode, concentration function.

\section{Introduction}

In BHT (= Bertin, Hengartner and Theodorescu (1981)), we gave a characterization of unimodal distribution functions and a representation theorem for the class of unimodal distribution functions, both in terms of their Lévy concentration functions. Discrete analogs of these results are given in the present paper. In order to avoid unnecessary lengths, we refer to BHT for the continuous case.

\section{Discrete distribution functions}

Let $\mathscr{F}_{\mathrm{d}}$ be the set of all discrete distribution functions $F$, i.e., $F$ is a nondecreasing function from $\mathbb{Z}=$ $\{\ldots,-1,0,1, \ldots\}$ into $[0,1]$ such that $\lim _{n \rightarrow-\infty} F(n)=0$ and $\lim _{n \rightarrow \infty} F(n)=1$. We define $F(-\infty)=0$ and $F(+\infty)=1$.

Further for $A \subset \overline{\mathbb{R}}=[-\infty, \infty]$, put $A_{\mathrm{d}}=A \cup \mathbb{Z} ;$ if $A$ is an interval, $A_{\mathrm{d}}$ is called a discrete interval. For a discrete interval $I$, Int $I=\left\{n \in I:[n-1, n+1]_{\mathrm{d}} \subset I\right\}$. Next, a function $G: I \rightarrow \mathbb{R}=(-\infty, \infty)$ is said to be convex at $n \in I$ if $n \notin \operatorname{Int} I$ or $G(n) \leqslant \frac{1}{2}(G(n+1)+G(n-1))$; in the case of strict inequality, $G$ is said to be strictly convex at $n \in I$. $G$ is said to be (strictly convex on $I$ whenever $G$ is (strictly) convex at each $n \in I$. Concavity is defined in a similar way, as well as affinity. If $\hat{I}$ denotes the obvious extension of the discrete interval $I$ to an interval of $\mathbb{R}$, then $\hat{G}$ denotes the obvious piecewise linear extension to $\hat{I}$ of a function $G: I \rightarrow \mathbb{R}$.

Work supported by the Natural Sciences and Engineering Research Council Canada Grant A-7223, by the Québec Action Concertee Grant ER-1023, and by the Deutsche Forschungsgemeinschaft. 
Let $I$ be a discrete interval of $\mathbb{R}$. For $G: I \rightarrow \mathbb{R}$ the following assertions are clearly equivalent:

(1) $G$ is convex on $I$;

(2) if $n \in$ Int $I$ then $G(n)-G(n-1) \leqslant G(n+1)-G(n)$;

(3) $G$ is the restriction to $I$ of a convex function $\bar{G}: \hat{I} \rightarrow \mathbb{R}$;

(4) $\hat{G}$ is convex on $\hat{I}$.

Let us note that $F \in \mathscr{F}_{\mathrm{d}}$ if and only if $\hat{F} \in \mathscr{F}$, where $\mathscr{F}$ is the set of all (right continuous) distribution functions on $\mathbb{R}$.

\section{Unimodal discrete distribution functions}

Let $F \in \mathscr{F _ { \mathrm { d } }} ; a \in \mathbb{Z}$ is called a mode of $F$ if and only if $F$ is convex on $[-\infty, a]_{\mathrm{d}}$ and concave on $[a, \infty)_{\mathrm{d}}$. Further $F$ is said to be unimodal if and only if $F$ has a mode. Let us denote by $\mathscr{U}_{\mathrm{d}}$ the set of all unimodal discrete distribution functions and by $\mathscr{U}_{\mathrm{d}, a}$ the set of those among them for which $a$ is a mode. Next $F \in \mathscr{F}_{\mathrm{d}}$ is said to be strictly unimodal if and only if $F$ has a mode $a, F$ is strictly convex on $\{x \in \mathbb{Z}: x \leqslant a$, $F(x+1)>0\}$, and $F$ is strictly concave on $\{x \in \mathbb{Z}: x \geqslant a, F(x-1)<1\}$. We shall indicate the strict unimodality by the superscript $\mathrm{s}$.

In what follows, if the subscript $\mathrm{d}$ does no appear, then the corresponding notations refer to $F \in \mathscr{F}$.

Theorem 3.1. Let $F \in \mathscr{F}_{\mathrm{d}}$. The following assertions are equivalent:

(a) $F \in \mathscr{U}_{\mathrm{d}}$;

(b) $\hat{F} \in \mathscr{U}(\mathscr{U}$ is the set of all unimodal $\mathrm{F} \in \mathscr{F})$;

(c) $G=F$ on $\mathbb{Z}$ for some $G \in \mathscr{U}$. Moreover, the set of modes of $F$ is the set of all integer modes of $\hat{F}$ and contains the set of all integer modes of $G$.

Proof. Let $a \in(n, n+1)$ be a mode of $G$ and suppose that neither $n$ nor $n+1$ is a mode of $F$. We have

$$
F(n)>2 F(n+1)-F(n+2) \text { and } F(n+1)<2 F(n)-F(n-1)
$$

and hence

$$
\begin{aligned}
G(a)-G_{-}(a) \leqslant & F(n+1)-(n+1-a) \times(F(n+2)-F(n+1))-F(n) \\
& -(a-n)(F(n)-F(n-1)) \\
< & (F(n)-F(n+1))(n+1-a+a-n-1)=0 .
\end{aligned}
$$

A similar result holds for strict unimodality:

Theorem 3.2. Let $F \in \mathscr{F}_{\mathrm{d}}$. The following assertions are equivalent:

(a) $F \in \mathscr{U}_{\mathrm{d}}^{s}$

(b) $G=F$ on $\mathbb{Z}$ for some strictly unimodal distribution function $G$ on $\mathbb{R}$.

Proof. Suppose that $F \in \mathscr{U}_{\mathrm{d} . a}^{s}$. Set

$$
p_{n}(x)=\frac{1}{2}(F(n+1)+F(n-1)-2 F(n))(x-n)^{2}+\frac{1}{2}(F(n+1)-F(n-1))(x-n)+F(n)
$$

for all $n \in \mathbb{Z}$. We have:

$(\alpha)$ if $n \leqslant a-1$ then $p_{n}$ is convex on $\mathbb{R}$ and nondecreasing on $[n, \infty)$ and if $n \geqslant a+1$ then $p_{n}$ is concave on $\mathbb{R}$ and nondecreasing on $(-\infty, n]$;

( $\beta$ ) if $n \leqslant a-1$ and if $F$ is strictly convex in $n$ then $p_{n}$ is strictly convex on $\mathbb{R}$ and if $n \geqslant a+1$ and if $F$ is strictly concave in $n$ then $p_{n}$ is strictly concave on $\mathbb{R}$; 
( $\gamma) F(n+i)=p_{n}(n+i), i=-1,0,1$.

Now let $\left\{p_{n}: n \in \mathbb{Z}\right\}$ be some family of continuous real valued functions with the properties $(\alpha),(\beta)$, and $(\gamma)$. Define

$$
G(x)= \begin{cases}\max \left\{p_{n}(x), p_{n+1}(x)\right\} & \text { for } n \leqslant x<n+1 \leqslant a-1, \\ p_{a-1}(x) & \text { for } a-1 \leqslant x<a \\ p_{a+1}(x) & \text { for } a \leqslant x<a+1 \\ \min \left\{p_{n}(x), p_{n+1}(x)\right\} & \text { for } a+1 \leqslant n \leqslant x<n+1\end{cases}
$$

It is easily shown that $G$ is a continuous distribution function extending $F$ to $\mathbb{R}$, and strictly unimodal at $a$. Thus (a) implies (b); the proof of the converse implication is omitted.

Let us remark that $G$ in Theorem 3.1 (c) and in Theorem 3.2 (b) can be chosen to be continuous and such that the set of modes of $F$ is the set of integer modes of $G$.

\section{Concentration functions and pointers}

Let $F \in \mathscr{F}$; ; by analogy with the case $F \in \mathscr{F}$, we set

$$
Q_{F}(l)= \begin{cases}0 & \text { for } l<0 \\ \sup \{F(x+l)-F(x): x \in \mathbb{Z}\} & \text { for } l \in \mathbb{N} .\end{cases}
$$

$Q_{F}$ is said to be the (Lévy) concentration function of $F$. As in the case $F \in \mathscr{F}$ (for details, see Hengartner and Theodorescu (1973)), the mapping $F \rightarrow Q_{F}$ is a continuous convex idempotent surjection from $\mathscr{F}_{\mathrm{d}}$ onto the set of all subadditive discrete distribution functions $G$ such that $G(0)=0$. Clearly:

Proposition 4.1. Let $l \in \mathbb{Z}$. Then $Q_{F}(l)=Q_{\hat{F}}(l)$.

Further, consider the pointer $A_{F}$ of $F$ defined by

$$
A_{F}(l)=\left\{x \in \mathbb{Z}: Q_{F}(l)=F(x+l)-F(x)\right\} \quad \text { for } l \in \mathbb{N} .
$$

For $l \in \mathbb{N}^{*}=\{1,2, \ldots\}$ each $A_{F}(l)$ is a finite nonempty set. Let $[x]$ denote the integral part of $x$.

Proposition 4.2. Let $l \in \mathbb{N}, \varepsilon \geqslant 0$, and $x \in A_{\hat{F}}(l+\varepsilon)$. Set $J=[[x],[x]+1-\varepsilon]$. Then

(a) $x-[x] \leqslant \varepsilon \leqslant 1-x+[x]$ implies

$$
J \subset A_{\hat{F}}(l+\varepsilon) \quad \text { or } \quad\{x\}=\{[x]\}=A_{\hat{F}}(l+\varepsilon) \cap J \quad \text { or } \quad\{x\}=\left\{[x]+1-\varepsilon=A_{\hat{F}}(l+\varepsilon) \cap J ;\right.
$$

(b) $A_{\hat{F}}(l)=A_{F}(l) \cup\left\{[n, n+1]: n, n+1 \in A_{F}(l)\right\}$;

(c) $A_{\hat{F}}(l) \cap \mathbb{Z}=A_{F}(l)$;

(d) if $A_{\digamma}(l)$ is a discrete interval, then $A_{\hat{F}}(l)$ is an interval.

Proof. Since the function $y \rightarrow \hat{F}(y+l+\varepsilon)-\hat{F}(v)$ is linear on $J$, it either has a maximum in some endpoint of $J$ or is constant on $J$.

Further, we have:

Theorem 4.3. $Q_{\hat{F}}$ is a piecewise linear distribution function on $\mathbb{R}$ and $Q_{\hat{f}}(l)=\alpha l$ on $[0,1]$, where $\alpha$ $=\max \{F(x+1)-F(x): x \in \mathbb{Z}\}$. 
Proof. The second statement is obvious. By Remark 2.1 of BHT $Q_{\hat{r}}$ is a continuous distribution function on $\mathbb{R}$. We have to show that, for each $l_{0}>0, Q_{\hat{F}}$ is affine on $\left[l_{0}-\varepsilon, l_{0}\right]$ and on $\left[l_{0}, l_{0}+\varepsilon\right]$ for some $\varepsilon>0$. Choose $l_{0}>0$. Let $0<\delta<\min \left(1,\left[l_{0}\right]+1-l_{0}\right)$ and $\delta<l_{0}-\left[l_{0}\right]$ if $l \neq\left[l_{0}\right]$. Since $A_{\hat{r}}$ is cocontinuous (cf. Theorem 2.3(3) of BHT, there exists $\varepsilon \in(0, \delta)$ such that, for $\left|l-l_{0}\right| \leqslant \varepsilon$ and $y \in A_{\hat{r}}(l)$, there exists $x \in A_{\hat{F}}\left(l_{0}\right)$ such that $|y-x|<\delta$ and $\left|y+l-x-l_{0}\right|<\delta$. By Proposition 4.2, either $A_{\hat{F}}(l) \cap \mathbb{Z} \neq 0$ or $\left(A_{\hat{F}}+I\right)(l) \cap \mathbb{Z} \neq 0$. We only consider the first case. Let

$$
y \in A_{\hat{r}}(l) \cap \mathbb{Z}, x \in A_{\hat{r}}\left(l_{0}\right), I_{0} \leqslant l \leqslant l_{0}+\varepsilon \text { and }|y-x|<\delta .
$$

Proposition 4.2(a) shows that $y \in A_{\hat{F}}\left(l_{0}\right)$. Hence

$$
\begin{aligned}
Q_{\hat{F}}(l) & =\hat{F}(y+l)-\hat{F}(y)=\hat{F}(y+l)-\hat{F}\left(y+l_{0}\right)+\hat{F}\left(y+l_{0}\right)-\hat{F}(y) \\
& =Q_{\hat{F}}\left(l_{0}\right)+\left(l-l_{0}\right)\left(F\left(y+\left[l_{0}\right]+1\right)-F\left(y+\left[l_{0}\right]\right)\right) . \quad \square
\end{aligned}
$$

In the sequel we shall need the following auxiliary lemmas:

Lemma 4.4. Let $F \in \mathscr{F}_{\mathrm{d}}, J=A_{F}\left\langle[1, \infty)_{\mathrm{d}}\right\rangle$, and $K=\left(A_{\digamma}+I\right)\left\langle[1, \infty)_{\mathrm{d}}\right\rangle$, where $I$ is the identity mapping. Then

(a) $F(\inf J)=0$;

(b) $F(\sup K)=1$;

(c) $1+\sup J \geqslant \inf K$.

Lemma 4.5. Let $l \in \mathbb{N}^{*}, x \in A_{F}(l)$, and $y \in A_{F}(l)+l$. Suppose that $Q_{F}$ is concave on $[l-1, l+1]_{\mathrm{d}}$. Then $F(x) \leqslant \frac{1}{2}[F(x+1)-F(x-1)]$ and $F(y) \geqslant \frac{1}{2}[F(y+1)-F(y-1)]$. Moreover, the inequalities are strict whenever $Q$ is strictly concave in 1 .

Proof. $F(x+l)-F(x)=Q_{F}(l) \geqslant \frac{1}{2}\left[Q_{F}(l+1)+Q_{F}(l-1)\right] \geqslant \frac{1}{2}[F(x+l)-F(x-1)]+\frac{1}{2}[F(x+l)$ $-F(x+1)]$ and hence

$$
F(x) \leqslant \frac{1}{2}[F(x+1)-F(x-1)] .
$$

Lemma 4.6. Let $Q_{F} \in \mathscr{U}_{\mathrm{d}}, l \in \mathbb{N}^{*}$, and $x, y \in A_{F}(l)$. If $Q_{F}$ is strictly concave at $l$ then either $x=y$ or $|x-y|>l$.

Proof. Suppose that $0<|x-y| \leqslant l$. Then

$$
Q_{F}(l)>\frac{1}{2} Q_{F}(x+l-y)+\frac{1}{2} Q_{F}(y+l-x) \geqslant \frac{1}{2} F(x+l)-\frac{1}{2} F(y)+\frac{1}{2} F(y+l)=Q_{F}(l) .
$$

We need the following auxiliary notions. Let $\phi$ be a real-valued function defined on a discrete interval $J$; $\alpha \in \mathbb{R}$ is said to be a subdifferential of $\phi$ at $x$ with respect to $J$ if and only if $\phi(y)-\phi(x) \geqslant \alpha(y-x)$ for each $y \in J$. The notion of superdifferential is defined in a dual way.

Lemma 4.7. Let $F \in \mathscr{F}_{\mathrm{d}}, l \in \mathbb{N}$, and $x \in A_{\hat{F}}(l)$. Then every superdifferential of $Q_{\hat{r}}$ at $l$ with respect to $\mathbb{N}$ is a subdifferential of $\hat{F}$ at $x$ with respect to $[-\infty, x+l]_{d}$ and a superdifferential of $\hat{F}$ at $x+l$ with respect to $[x, \infty]_{d}$.

Proof. Indeed,

$$
\alpha(k-l) \geqslant Q_{\hat{r}}(k)-Q_{\hat{r}}(l) \geqslant F(x+l)-F(x+l-k)-F(x+l)+F(x)=F(x)-F(x+l-k)
$$

and

$$
\alpha(k-l) \geqslant Q_{\hat{F}}(k)-Q_{\hat{F}}(l) \geqslant F(x+k)-F(x)-F(x+l)+F(x)=F(x+k)-F(x+l),
$$

for each $k \in \mathbb{N}$ and each superdifferential $\alpha$ of $Q_{\hat{\kappa}}$ at $l$. 


\section{Main result}

In the sequel, a correspondence $A: \mathbb{N}^{*} \rightarrow \mathbb{Z}$ will be said to be $d$-cocontinuous if and only if $k, l \in \mathbb{N}^{*}$. $|k-l| \leqslant 1$ implies that for each $y \in A(l)$ there exists $x \in A(k)$ such that $|y-x| \leqslant 1$. The image under a $\mathrm{d}$-cocontinuous $\mathrm{A}$ of a discrete interval is again a discrete interval if each $A(l)$ is a discrete interval.

Our main result is contained in the next theorem:

Theorem 5.1. Let $F \in \mathscr{F}_{\mathrm{d}}$. Then $F \in \mathscr{U}_{\mathrm{d}}$ if and only if $Q_{F} \in \mathscr{U}_{\mathrm{d}}, A_{F}(l)$ is a discrete interval for each $l \in \mathbb{N} *$. and $A_{F}$ is d-cocontinuous.

Proof. Let $F \in \mathscr{U}_{\mathrm{d}}$, Then by Theorem 3.1(b) we have $\hat{F} \in \mathscr{U}$, hence, by Theorem 3.5 of BHT, $Q_{\hat{r}} \in \mathscr{U}$. It follows from Theorem 3.1(c) and Proposition 4.1 that $Q_{F} \in \mathscr{U}_{\mathrm{d}}$.

Next, again by Theorem 3.5 of BHT, each $A_{\hat{F}}(l)$ is an interval, hence. by Proposition 4.2(c), each $A_{f}(l)$ is a discrete interval.

Finally, let $b(l)=\inf A_{\hat{F}}(l)$ and $t(l)=\sup A_{\hat{F}}(l)$ be the larboard and starboard functions of $\hat{F}$, as defined in BHT. By Theorem 4.2 of BHT we have

$$
b(l) \leqslant b(k) \leqslant b(l)+l-k \text { and } t(l) \leqslant t(k) \leqslant t(l)+l-k
$$

whenever $k \leqslant l$. By Proposition 4.2(c), this implies the d-cocontinuity of $A_{F}$.

Suppose now that $Q_{F}$ and $A_{F}$ have the stated properties. The images $J=A_{F}\left\langle[1, \infty)_{\mathrm{d}}\right\rangle$ and $K=$ $\left(A_{F}+I\right)\left\langle[1, \infty)_{\mathrm{d}}\right\rangle$ are discrete intervals. By Lemma $4.5, F$ is convex on [inf $\left.J, \sup J+1\right]_{\mathrm{d}}$ and concave on [inf $K-1, \sup K]_{\mathrm{d}}$. Further, by Lemma $4.4(\mathrm{a}, \mathrm{b}), F$ is convex on $[-\infty \text {, sup } J+1]_{\mathrm{d}}$ and concave on [inf $K-1, \infty]_{\mathrm{d}}$. Finally $F \in \mathscr{U}_{\mathrm{d}}$ by Lemma 4.4 .

Let us remark that the condition of d-cocontinuity of $A_{F}$ in Theorem 5.1 may be replaced by the apparently weaker condition that both $A_{F}\left\langle[1, \infty)_{\mathrm{d}}\right\rangle$ and $\left(A_{F}+I\right)\left\langle[1, \infty)_{\mathrm{d}}\right\rangle$ are discrete intervals.

Corollary 5.2. Let $F \in \mathscr{U}_{\mathrm{d}}$. Then $Q_{\hat{F}}=\left(Q_{F}\right)^{\hat{n}}$.

Proof. By Theorem 4.3 it suffices to prove that $Q_{\hat{F}}$ is linear on each interval $[k, k+1], k \in \mathbb{N} *$. Suppose that $Q_{\hat{F}}$ has a crack at $l \in(k, k+1)$. Since $Q_{\hat{r}}$ is unimodal, it has two different superdifferentials $\alpha$ and $\beta$ at $l$ with respect to $\mathbb{N}$. Choose now $x \in A_{\hat{F}}(l)$. From Lemma 4.7 it follows that $\hat{F}$ has a convex crack at $x$ and a concave crack at $x+l$. But $\{x, x+l\} \not \subset \mathbb{Z}$.

Corollary 5.3. Let $F \in \mathscr{U}_{\mathrm{d}}$ and let $f$ be a nonincreasing selection of the correspondence $A_{F}$ such that $f(l)-f(l+1) \leqslant 1$. Then $\hat{f}$ is a selection of $A_{\hat{F}}$.

Proof. Let $l \geqslant 0, \lambda \in[0,1], x=f(l)$ and $x-1=f(l+1)$. By Corollary 5.2,

$$
\begin{aligned}
Q_{\hat{F}}(l+1-\lambda) & =\lambda Q_{F}(l)+(1-\lambda) Q_{F}(l+1) \\
& =\lambda F(x+l)-\lambda F(x)+(1-\lambda) F(x+l)-(1-\lambda) F(x-1) \\
& =F(x+l)-F(x+\lambda-1),
\end{aligned}
$$

and hence $\lambda f(l)+(1-\lambda) f(l+1) \in A_{\hat{F}}(l+1-\lambda)$. The assertion for the case $f(l+1)=x$ is proved in a similar way.

One of the main results of BHT (Theorem 3.5) says that $F \in \mathscr{U}$ if and only if $Q_{F} \in \mathscr{U}$ and $A_{F}(l)$ is an interval for each $l>0$. The following example shows that in the discrete case the condition that $A_{F}$ is $\mathrm{d}$-cocontinuous cannot be omitted. Moreover, the same example shows that the assertions of Corollaries 5.2 
and 5.3 are not valid for $F \notin \mathscr{U}_{\mathrm{d}}$ even if $Q_{F} \in \mathscr{U}_{\mathrm{d}}$. Indeed, take values as in Table 1 , with $0<\varepsilon<\frac{1}{6}$. Note that $Q_{\hat{F}}(1+4 \varepsilon /(1+4 \varepsilon))=\frac{1}{3}<\hat{Q}_{F}(1+4 \varepsilon /(1+4 \varepsilon))$ and that $A_{\hat{F}}(l)=\{-1-l\} \not \subset\left\{\hat{A}_{F}(l)\right\}$ for $4 \varepsilon /(1+$ $4 \varepsilon)<l<2$. See however Theorem 6.2.

\section{Consequences of the main result}

Let us note that the set of modes of $F \in \mathscr{U}_{\mathrm{d}}$ is a nonempty finite discrete interval $\left[a_{-}, a_{+}\right]_{\mathrm{d}}$. From Theorem 3.1, from Proposition 4.2(c) and from Corollary 3.3 of BHT, it follows immediately that for $l \in \mathbb{N}^{*}, A_{F}(l) \subset\left[a_{-}-l, a_{+}\right]_{\mathrm{d}}$ and that $A_{F}(l)$ is the set of all maximum points of the concave function $F(\cdot+l)-F(\cdot)$ on this interval. Next, from Corollary 5.2 and Theorem 5.4 of BHT, we obtain straightforwardly

Proposition 6.1. Let $F \in \mathscr{U}_{\mathrm{d}}$ and $\gamma \in \mathbb{N}^{*}$. Then $Q_{F}$ is affine on $[0, \gamma]_{\mathrm{d}}$ if and only if $a_{+}-a_{-} \geqslant \gamma$. Moreover, the slope of $Q_{\hat{F}}$ on $[0, \gamma]_{d}$ equals the slope of $\hat{F}$ on $\left[a_{-}, a_{+}\right]$.

From Proposition 5.1 it follows that $a_{-}<a_{+}$, i.e., $F \in \mathscr{U}_{\mathrm{d}}$ has at least two modes.

The next application of Theorem 5.1 is the discrete analogon of the Theorem of Hengartner and Theodorescu (1978) and Corollary 3.6 of BHT:

Theorem 6.2. Let $F \in \mathscr{F}_{\mathrm{d}}$. Then $F \in \mathscr{U}_{\mathrm{d}}^{s}$ if and only if $Q_{F} \in \mathscr{U}_{d}^{s}$.

Proof. Let $F \in \mathscr{U}_{\mathrm{d}}^{s}$ and $l \in \mathbb{N}$ such that $0<Q_{F}(l)<1$. Choose $x \in A_{F}(l)$. We have

$$
1>Q_{F}(l)=F(x+l)-F(x)>\frac{1}{2} F(x+l+1)+F(x+l-1)-F(x) \geqslant \frac{1}{2} Q_{F}(l-1)+Q_{F}(l+1)
$$

and hence $Q_{F} \in \mathscr{U}_{\mathrm{d}}^{s}$.

Conversely, suppose that $Q_{F} \in \mathscr{U}_{\mathrm{d}}^{s}$ and that $L=\sup \left\{l: Q_{F}(l)<1\right\} \geqslant 2$. Notice that $Q_{F}(l)>\frac{2}{3}$ for some $l \in[2, L]$ and that

$$
y+l-1 \geqslant x \text { and } y \leqslant x-l \text { if } x \in A_{F}(l), y \in A_{f}(l-1) \text { and } Q_{F}(l)>\frac{2}{3} .
$$

Moreover, for $2 \leqslant l \leqslant L, y<x \leqslant y+l-1$ would imply the contradiction

$$
\begin{aligned}
Q_{F}(l-1)+Q_{F}(l) & >Q_{F}(l+x-y)+Q_{F}(y+l-1-x) \\
& \geqslant F(l+x)-F(y)+F(y+l-1)-F(x) \\
& \geqslant Q_{F}(l)+Q_{F}(l-1) .
\end{aligned}
$$

\begin{tabular}{|c|c|c|c|c|}
\hline$x$ & $F(x)$ & 1 & $A_{F}(l)$ & $Q_{F}(l)$ \\
\hline-4 & 0 & 1 & $\{0\}$ & 1 \\
\hline-3 & $\frac{1}{6}$ & 2 & $\{-3\}$ & $\frac{1}{2}$ \\
\hline-2 & $\frac{1}{3}+\frac{2}{3} \varepsilon$ & 3 & $\{-4\}$ & $\stackrel{2}{3}$ \\
\hline-1 & $\frac{2}{3}$ & 4 & $\{-3\}$ & $\begin{array}{l}5 \\
6\end{array}$ \\
\hline 0 & $\frac{2}{3}$ & 5 & $\{-4\}$ & 1 \\
\hline 1 & 1 & 6 & $\{-5,-4\}$ & 1 \\
\hline
\end{tabular}

Table 1 
Similarly, $\mathrm{y}>x+l$ or $y-1 \leqslant x$. Consequently,

$$
x \leqslant y \leqslant y+l-1 \leqslant x+l \text { if } Q_{F}(l)>\frac{2}{3} .
$$

Now let (2) be satisfied for $l \in I=\left[l_{0}, L\right]_{\mathrm{d}} \cap \mathbb{N}, l_{0} \geqslant 2$.

Since $A_{F}\langle I\rangle$ is a discrete interval, it follows from Lemma 4.5, that (1) holds, and hence also (2), for $l=l_{0}-1>1$. This implies $l_{0}=2, F \in \mathscr{U}_{\mathrm{d}}$ by Theorem 5.1, and $F \in \mathscr{U}_{\mathrm{d}}^{s}$ by Lemma 4.5 .

The explicit relation between a unimodal distribution function, its pointer and its concentration function is contained in the following representation theorem:

Theorem 6.3. (1) Let $F$ be a numerical function on $\mathbb{Z}$ and $a \in \mathbb{R}$. We have $F \in \mathscr{U}_{\mathrm{d} . a}$ if and only if $F$ is of the form

$$
F(x)= \begin{cases}\sum_{l=f^{\prime}(x)}^{\infty}(Q(l+1)-Q(l))(f(l)-f(l+1)) & \text { for } x \leqslant a, \\ Q g^{i}(x)+F f g^{i}(x) & \text { for } x \geqslant a,\end{cases}
$$

where

(i) $Q \in \mathscr{U}_{\mathrm{d} .0}$ is a subadditive function;

(ii) $f$ is a nonincreasing function such that $f(0)=a$ and $g=f+I$ is nondecreasing;

(iii) $\Sigma(Q(l+1)-Q(l))(f(l)-f(l+1))<\infty$;

(iv) $f^{s}(x)=\inf f^{-1}(x), g^{i}(x)=\sup g^{-1}(x)$.

(2) In this case, $f$ is necessarily a selection of $A_{F}, Q=Q_{F}$ and one may choose $f(1)=\inf A_{F}(1)$ or $f(1)=\sup A_{F}(1)$.

Proof. This is a translation of Theorem 6.2 of BHT, by means of Theorem 3.1, Proposition 4.2 and Corollary 5.2 .

\section{Final remark}

Let $F \in \mathscr{F}_{\mathrm{d}}$; according to Keilson and Gerber (1971), $a \in \mathbb{Z}$ is called a mode of $F$ if and only if $F$ is convex on $[-\infty, a]_{\mathrm{d}}$ and concave on $[a-1, \infty)_{\mathrm{d}}$. Further $F$ is said to be unimodal if and only if $F$ has a mode. Let us denote by $\overline{\mathscr{U}}_{\mathrm{d}}$ the set of all unimodal $F \in \mathscr{F}_{\mathrm{d}}$ and by $\overline{\mathscr{U}}_{\mathrm{d}, a}$ the set of those among them for which $a$ is a mode. Clearly

$$
\overline{\mathscr{U}}_{\mathrm{d}}=\mathscr{U}_{\mathrm{d}}, \quad \overline{\mathscr{U}}_{\mathrm{d}, a}=\mathscr{U}_{\mathrm{d}, a-1} \cap \mathscr{U}_{\mathrm{d}, a} \quad \text { and } \quad \mathscr{U}_{\mathrm{d}, a-1}=\overline{\mathscr{U}}_{\mathrm{d}, a-1} \cup \overline{\mathscr{U}}_{\mathrm{d}, a} ;
$$

consequently, our main results, Theorems 5.1 and 6.2 , hold also, up to obvious modifications, for this type of discrete unimodality. The above mentioned definition of a mode fits better to the intuitive idea of a maximum at $a$ for the probability density. However, it can be shown that $\mathscr{U}_{\text {d. } a}$ has a nicer extreme boundary; as a result, $\mathscr{U}_{\mathrm{d} . a}$ is better adapted to the application of Choquet theory and admits unique representations of Hinčin type. This is the main reason for introducing in this paper a more general definition of a mode. 


\section{References}

Bertin, E.M.J., W. Hengartner and R. Theodorescu (1981), Some characterizations of unimodal distribution functions, Z. Wahrsch. Verw. Gebiete 57, 327-338.

Bertin, E.M.J. and R. Theodorescu (1981), Characterizations of unimodal distribution functions, in: D. Dugué, E. Lukacs and V.K. Rohatgi, eds., Analytical Methods in Probability Theory, Oberwolfach 1980, Lecture Notes in Mathematics 861 (Springer, Berlin), pp. 10-14.
Hengartner, W. and R. Theodorescu (1973), Concentration functions (Academic Press, New York; revised Russian version (1980): Nauka, Moscow).

Hengartner, W. and R. Theodorescu (1978), A characterization of strictly unimodal distribution functions by their concentration functions, Publ. Inst. Statist. Paris. 24, 1-10.

Keilson, J. and H. Gerber (1971), Some results for discrete unimodality, J. Amer. Statist. Assoc. 66, 386-389. 